\title{
Efecto del Ultrasonido de Baja Intensidad (USBI) sobre el Movimiento Dentario Ortodóncico. Estudio in vivo en Ratas Sprague-Dawley
}

\author{
Effect of Low Intensity Ultrasound (USBI) in the Orthodontic \\ Dental Movement. In vivo Study in Sprague-Dawley Rats
}

Victor Rojas ${ }^{1}$; Alejandra Nineham ${ }^{2}$; Cristobal Gajardo ${ }^{2}$; Adrián Rodriguez ${ }^{2}$ \& Rodrigo Oyonarte Weldt ${ }^{1}$

ROJAS, V.; NINEHAM, A.; GAJARDO, C.; RODRIGUEZ, A. \& OYONARTE, W. R. Efecto del ultrasonido de baja intensidad (USBI) sobre el movimiento dentario ortodóncico. Estudio in vivo en ratas Sprague-Dawley. Int. J. Morphol., 38(1):101-108, 2020.

RESUMEN: Este estudio se realizó con el propósito de analizar el efecto de la aplicación de ondas de USBI sobre la velocidad y distancia de movimiento dentario, y su efecto a nivel histológico en ratas Sprague-Dawley. Veintitrés ratas macho Sprague-Dawley fueron parte del experimento, las que fueron divididas aleatoriamente en tres grupos. Cinco ratas fueron utilizadas como control sin movimiento dentario ortodóncico (MDO), nueve ratas fueron sometidas a una aplicación de fuerza ortodóncica sin aplicación de USBI y nueve fueron sometidas a una aplicación de fuerza ortodóncica con USBI. Estas fuerzas ortodóncicas fueron realizadas entre el aspecto mesio vestibular del primer molar superior izquierdo y el incisivo central superior, traccionando el molar hacia mesial, aplicando una fuerza aproximada de 50 gramos durante 21 días, debido a problemas con la sedación estos dos últimos grupos quedaron compuestos de seis y ocho ratas respectivamente. En el grupo MDO sin USBI, se registró una reducción del espacio entre molares e incisivos de 2 mm. En el grupo MDO con USBI dicha reducción fue de $3.4 \mathrm{~mm}$, siendo esta diferencia estadísticamente significativa. Histológicamente el grupo MDO c/USBI mostró un perímetro vascular y espacios medulares aumentados respecto de los otros grupos, no obstante, no se encontraron diferencias significativas en la cantidad de osteoclastos. Fue posible observar y demostrar cuantitativamente que el USBI es una forma de estimulación mecánica eficiente para la aceleración del MDO sobre un período experimental de 21 días, donde se observaron diferencias significativas entre el grupo control, el grupo MDO sin USBI y el grupo MDO con USBI en la magnitud del movimiento dentario. Se observó una mayor vascularidad en el grupo con USBI, expresando perímetros vasculares de mayor tamaño, esto pudo deberse al efecto estimulatorio del ultrasonido sobre la angiogénesis.

PALABRAS CLAVE: Ultrasonido de baja intensidad; Movimiento dentario.

\section{INTRODUCCIÓN}

El tiempo total de un tratamiento de ortodoncia es un factor de alta relevancia clínica tanto para el paciente como para el ortodoncista. Debido a esto, en las últimas décadas, se han estudiado nuevas técnicas tanto quirúrgicas como no quirúrgicas, para acelerar el movimiento dentario ortodóncico con el fin de disminuir los tiempos de tratamiento, reducir efectos adversos como el dolor, disconfort del paciente y daño periodontal. La aceleración del movimiento dentario y el acortamiento de un tratamiento ortodóncico podría lograr en algunos casos, minimizar daños como la reabsorción radicular y la pérdida de vitalidad pulpar (Zainal Ariffin et al., 2011; Huang et al., 2014).

El movimiento dentario ortodóncico (MDO) es el resultado de una respuesta biológica a una interferencia en el equilibrio fisiológico del complejo dentofacial debido a la aplicación de una fuerza externa (Proffit, 2013). Se caracteriza por una remodelación en los tejidos dentales y periodontales, donde la fuerza aplicada sobre el diente genera zonas de compresión y tensión en el hueso alveolar y en el ligamento periodontal (Masella \& Meister, 2006). Se ha sugerido que se puede incrementar la actividad celular del ligamento periodontal con métodos que complementan la aplicación de fuerzas mecánicas, y que se podrían usar en combinación con estas últimas para una remodelación ósea más rápida (Kale et al., 2004).

Factores como la edad, presencia de enfermedades sistémicas y consumo de fármacos son factores intrínsecos, propios del paciente, que pueden afectar el metabolismo

\footnotetext{
${ }^{1}$ Departamento de Ortodoncia, Facultad Odontología Universidad de los Andes, Santiago, Chile.

${ }^{2}$ Práctica privada, Santiago, Chile.
} 
ROJAS, V.; NINEHAM, A.; GAJARDO, C.; RODRIGUEZ, A. \& OYONARTE, W. R. Efecto del ultrasonido de baja intensidad (USBI) sobre el movimiento dentario ortodóncico. Estudio in vivo en ratas Sprague-Dawley. Int. J. Morphol., 38(1):101-108, 2020.

óseo. Además, existen factores extrínsecos ampliamente investigados en animales y humanos que pueden modificar el metabolismo óseo con el fin de estimular el MDO (Tyrovola \& Spyropoulos, 2001).

Cuando el tejido óseo y sus estructuras periodontales adyacentes son expuestos a cargas mecánicas en diferentes grados de magnitud, frecuencia y duración, sufren extensos cambios macroscópicos y microscópicos. Asimismo, las cargas mecánicas también pueden modificar el flujo sanguíneo del tejido periodontal, resultando en la síntesis y liberación local de varias moléculas tales como, neurotransmisores, citoquinas, factores de crecimiento, factores estimuladores de colonias. Las moléculas liberadas provocan una respuesta celular en el diente y los tejidos que lo rodean, generando un microambiente favorable para la aposición o reabsorción de tejido óseo, activándose múltiples vías de señales celulares, donde finalmente se habrá estimulado al ligamento periodontal, y al mecanismo de reabsorción/aposición localizada de hueso (Patil et al., 2013).

El ultrasonido, es una onda de presión acústica a frecuencias sobre el límite de audición humana, que es transmitida a través, y hacia el interior de los tejidos biológicos. Este ha sido ampliamente utilizado en medicina como una herramienta terapéutica, operativa y diagnóstica (El-Bialy et al., 2004). El ultrasonido pulsátil de baja intensidad (USBI), por su parte, es una forma de estimulación mecánica celular que ha demostrado ser eficiente incrementando la expresión del factor de crecimiento vascular endotelial (VEGF) (Lu et al., 1999) y a la vez de estimular la diferenciación de células mesenquimales en cultivos celulares (Becerra et al., 2012). Incrementa la actividad osteoblástica, el flujo sanguíneo local y la velocidad de aposición mineral en el proceso de reparación de fracturas en humanos, siendo todos estos fenómenos favorables para la eventual aceleración del movimiento dentario (Rutten et al., 2008).

El-Bialy et al. (2003), reportaron efectos de la aplicación de USBI sobre incisivos de conejo, demostrando un incremento en el crecimiento radicular y aceleración de la erupción de los incisivos. En otro estudio realizado en premolares humanos sometidos a fuerzas ortodóncicas y tratados con USBI (El-Bialy et al., 2004) que fueron extraídos posteriormente por indicación ortodóncica, se observó que presentaban menor reabsorción radicular, gracias a un proceso reparativo mediado por la aposición de cemento.

Dados los efectos previamente descritos de la aplicación de USBI, la ejecución de movimientos dentarios ortodóncicos (MDO), en combinación con estímulos físicos como la aplicación de USBI permitiría establecer un medio propicio para la optimización del MDO en términos de aumentar su velocidad, reducir la sintomatología dolorosa y complicaciones derivadas de la reabsorción radicular.

El presente estudio se realizó con el objetivo de analizar el efecto de la aplicación de ondas de USBI sobre la velocidad de movimiento dentario en ratas Sprague-Dawley cuantificado clínicamente e histológicamente a nivel óseo alveolar.

\section{MATERIAL Y MÉTODO}

Después de la revisión y aceptación del protocolo de investigación por parte de la comisión de investigación de la Facultad de Odontología de la Universidad de Los Andes, 23 ratas macho Sprague-Dawley de entre 250 a $300 \mathrm{~g}$ de peso fueron incluidas en el estudio. Se realizaron procedimientos de movilización ortodóncica entre el aspecto el primer molar superior izquierdo y el incisivo central superior, traccionando el molar hacia mesial, aplicando una fuerza compresiva de aproximadamente 50 gramos durante 21 días. Las ratas fueron divididas aleatoriamente en tres grupos. Cinco ratas fueron utilizadas como control sin movimiento dentario (Control sin MDO), nueve ratas fueron sometidas a una aplicación de fuerza ortodóncica sin USBI (MDO s/ USBI) y nueve fueron sometidas a una aplicación de fuerza ortodóncica con USBI (MDO c/USBI).

Diseño Experimental. A las ratas de los grupos MDO s/ USBI y MDO c/USBI, se les instaló la aparatología ortodóncica para la mesialización del primer molar superior izquierdo, usando resortes de Niquel-Titanio (NiTi) superelástico de $5 \mathrm{~mm}$ de longitud (Sentalloy, GAC Int. NY, USA), fijados con ligaduras de acero inoxidable de 0,008" tanto en el incisivo como en el molar, reforzados con cemento de resina Transbond XT Light Cure Adhesive (3M Unitek, Ca., USA) (Fig. 1A). Los incisivos superiores fueron ferulizados, los incisivos inferiores y primer molar inferior se desgastaron en sus márgenes incisales y superficies oclusales respectivamente. Los aparatos se instalaron bajo anestesia general de administración intraperitoneal de Ketamina y Xilacina, inyectada en una proporción de 9:1 en una dosis de $1 \mu \mathrm{l} / \mathrm{Kg}$.

Ultrasonido. Las ondas de Ultrasonido de Baja Intensidad fueron aplicadas sobre la piel que cubre la zona maxilar que aloja al primer molar superior izquierdo con abundante gel sónico empleando un posicionador de silicona para el transductor (Fig. 1B y 1C). La aplicación de ondas USBI se realizó bajo sedación con de 0,3 mL de Ketamina y 0,03 mL de Xilacina. La intensidad de emisión de US fue de $0.05 \mathrm{~W} /$ $\mathrm{cm}^{2}$ (1MHz, pulsos de 200 microsegundos), en sesiones dia- 

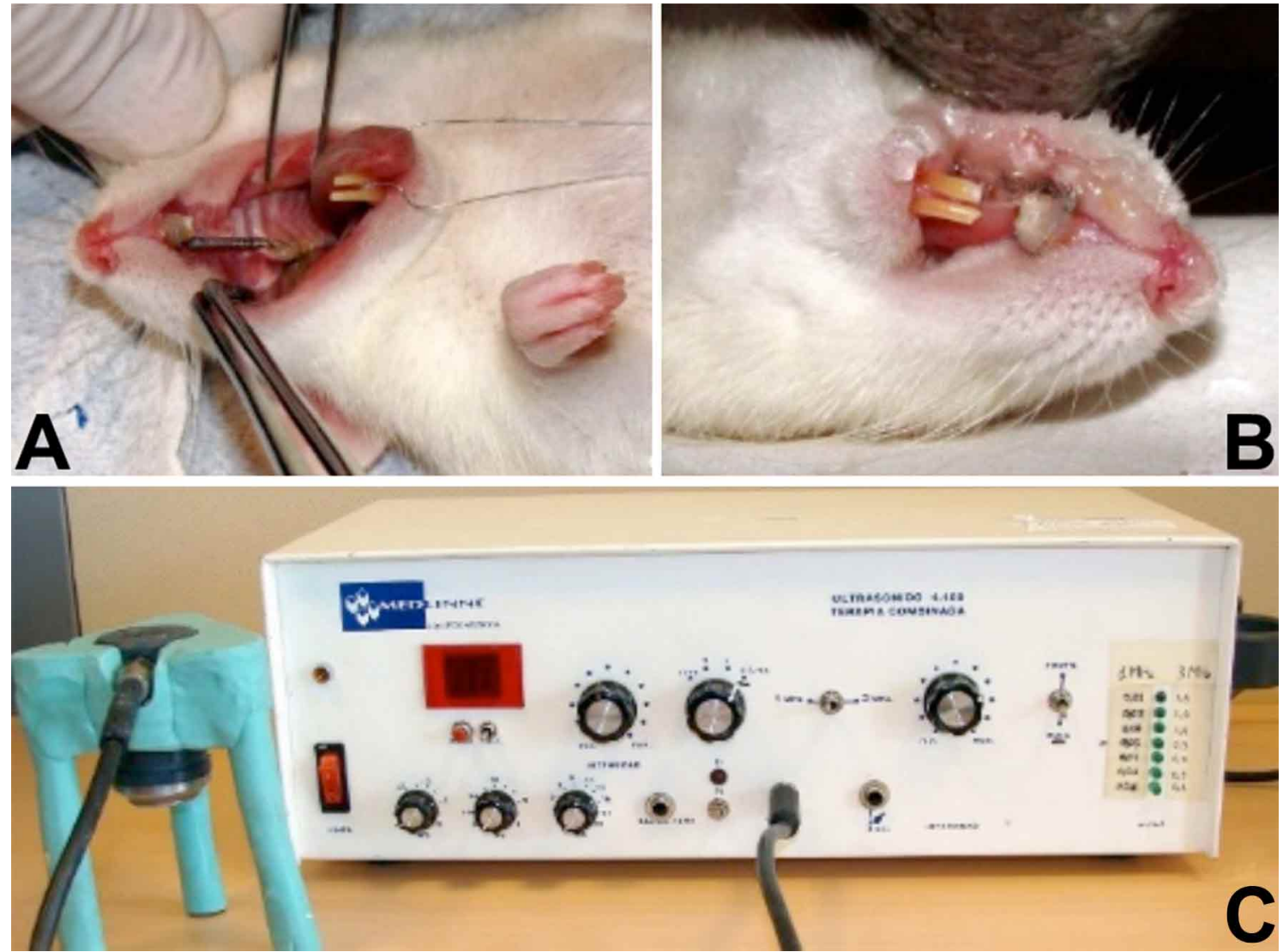

Fig. 1. (A) Aparatología ortodóncica instalada. (B) Estimulación con USBI. (C) Aparato de USBI.

rias de 20 minutos de duración por un total de 21 días consecutivos. Al finalizar el experimento, las ratas fueron sacrificadas con una sobredosis de éter.

Movimiento Dentario. El movimiento dentario fue medido utilizando un pie de metro digital (S.Tools, modelo S406755 0-100 mm Digital Caliper, China), realizando registros diarios de distancia en milímetros, con una sensibilidad de $0.01 \mathrm{~mm}$, entre los márgenes gingivales del aspecto más mesial del 1er molar superior izquierdo y el aspecto más distal del incisivo ipsilateral. En cada sesión de medición se realizaron 3 registros, tanto en el lado ipsilateral a la tracción ortodóncica como contralateral, utilizando el promedio de cada lado para fines analíticos.

Análisis Histológico. Los maxilares fueron fijados en formalina (10\%), descalcificados en solución Ana Morse, impregnados en parafina y seccionados en cortes de $5 \mu$, para luego aplicar tinción hematoxilina-eosina (H-E), y fosfatasa ácida tartrato resistente (TRAP), tinción para la identificación de osteoclastos. Las imágenes histológicas fueron obtenidas con un microscopio óptico (Nikon Labophot, Japón) y fotografiadas con una cámara digital (Nikon Coolpix L11) con una magnificación de 4x y 40x.
Análisis Cualitativo. La evaluación cualitativa se enfocó en la descripción de las características de vasos sanguíneos, la descripción de los espacios medulares y presencia de osteoclastos en los distintos campos de evaluación en cada muestra histológica.

Análisis Cuantitativo. Realizamos un análisis histomorfométrico utilizando el programa Sigmascan Pro 5.0 (SPSS Science, Chicago Ill.). En relación a la vascularidad se demarcó el perímetro vascular en cuatro zonas de la raíz mesial (RM) del primer molar superior izquierdo; mesio coronal (MC), mesio apical (MA), disto coronal (DC) y disto apical (DA), mientras que en la raíz distal (RD) fueron dos zonas; disto coronal (DC) y disto apical (DA), cada una de las zonas principales fue subdividida para abarcar tejido dentario-ligamento periodontal (LD) y ligamento periodontal-hueso alveolar (LH). Los espacios medulares fueron medidos en su perímetro las mismas 6 zonas anteriormente descritas. Los osteoclastos fueron cuantificados en dos zonas de la raíz mesial; mesio coronal (MC) y mesio apical (MA), mientras que en la raíz distal fue cuantificado en la zona disto coronal (DC).

Análisis Estadístico. Los datos fueron analizados con el software STATA versión 10.1. Para evaluar la normalidad de la 
ROJAS, V.; NINEHAM, A.; GAJARDO, C.; RODRIGUEZ, A. \& OYONARTE, W. R. Efecto del ultrasonido de baja intensidad (USBI) sobre el movimiento dentario ortodóncico. Estudio in vivo en ratas Sprague-Dawley. Int. J. Morphol., 38(1):101-108, 2020.

distribución de las variables histológicas, se utilizó el test de Shapiro-Wilk. Las variables que resultaron tener distribución normal se describieron y compararon a través de sus medias y desviaciones estándar. En cambio, las variables que no presentan una distribución normal se describieron y compararon utilizando la mediana y el rango intercuartílico. Las medidas individuales del movimiento dentario fueron descritas por el promedio y desviación estándar. Las diferencias entre los grupos experimentales de la respuesta en un determinado tiempo fueron comparadas a través de un análisis de varianza (ANOVA) con el test de comparaciones múltiples de Scheffé. Los resultados histológicos fueron analizados utilizando la prueba de Kruskal-Wallis. Para la comparación entre los grupos de estudio se empleó la corrección de Bonferroni.

\section{RESULTADOS}

Cuatro ratas fallecieron debido a una sobredosis de Ketamina y Xilacina, quedando en total 19 ratas. Los grupos experimentales incluyeron finalmente 8 ratas MDO c/ USBI y 6 ratas MDO s/USBI. El grupo control sin MDO permaneció con 5 ratas.

Tres ratas de cada grupo fueron escogidas aleatoriamente para realizar el análisis histológico.

Movimiento Dentario. En el grupo control sin MDO, la distancia interdentaria (entre primeros molares superiores e incisivos superiores) tanto en el lado derecho como izquierdo no presentó diferencias significativas, por otro lado, en los grupos MDO s/USBI y MDO c/USBI el lado derecho se mantiene relativamente constante, mientras que en el lado izquierdo (lado tratado) disminuye la distancia interdentaria. Al día 21, el grupo control sin MDO presentó un aumento de desplazamiento promedio de $0.1 \mathrm{~mm}$, en el grupo MDO s/USBI la distancia disminuyó $2 \mathrm{~mm}$ y en el grupo MDO c/ USBI la disminución fue de $3.4 \mathrm{~mm}$, existiendo diferencias intergrupales estadísticamente significativas (ANOVA p< 0,001). En la Tabla I se comparan los promedios de las diferencias de distancia entre los grupos, los cuales resultan ser estadísticamente significativos.

Tabla I. Diferencia entre grupos analizados (Scheffé $\mathrm{p}<0,001$ )

\begin{tabular}{llll}
\hline $\begin{array}{l}\text { Diferencias entre } \\
\text { grupos }\end{array}$ & $\begin{array}{l}\text { Control sin } \\
\text { MDO }-\end{array}$ & $\begin{array}{l}\text { Control sin } \\
\text { MDO }-\end{array}$ & $\begin{array}{l}\text { MDO } \\
\text { s/USBI }-\end{array}$ \\
& $\begin{array}{l}\text { MDO } \\
\text { s/USBI }\end{array}$ & $\begin{array}{l}\text { MDO } \\
\text { c/USBI }\end{array}$ & $\begin{array}{l}\text { MDO } \\
\text { c/USBI }\end{array}$ \\
\hline $\begin{array}{l}\text { Distancia promedio } \\
(\mathrm{mm})\end{array}$ & $1.93^{*}$ & $3.29^{*}$ & $1.35^{*}$ \\
\hline
\end{tabular}

En los grupos MDO s/USBI y MDO c/USBI se observa una fase inicial movimiento entre los días 0 y 4 , con un mayor movimiento en el grupo MDO c/USBI, posteriormente se observa una fase de latencia evidente entre los días 4 y 10. En el grupo MDO c/USBI, se observa una fase de movimiento progresivo más acentuada desde el día 10 en adelante, la cual se observa también partir del día 10 en el grupo MDO s/USBI. La curva de movimiento dentario se presenta en la Figura 2.

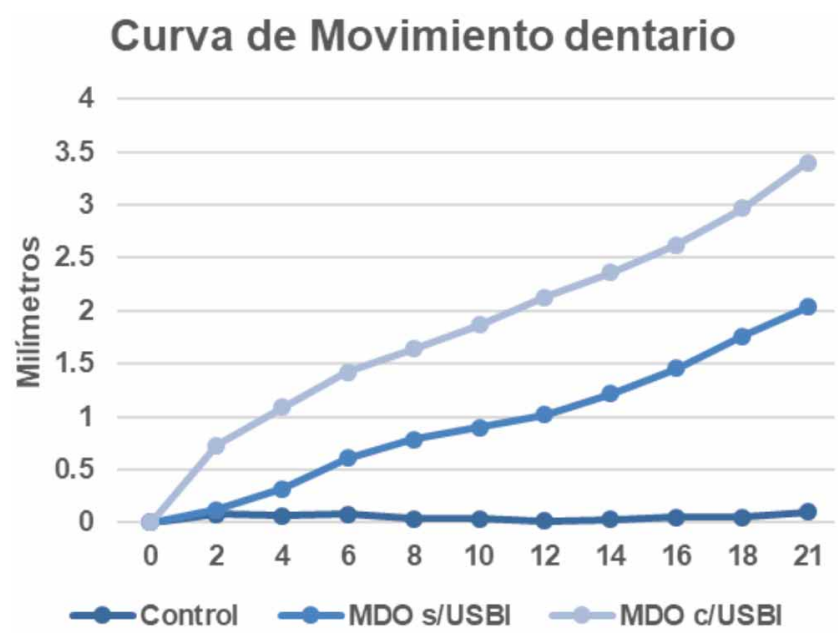

Fig. 2. Gráfico de curva de movimiento dentario

Análisis Histológico Cualitativo. Respecto a los vasos sanguíneos, estos se observaron de mayor perímetro y grosor en el grupo MDO c/ USBI respecto al grupo control sin MDO y MDO s/USBI (Fig. 3). Los grupos MDO s/USBI y MDO c/USBI, mostraron cambios histológicos a nivel del hueso alveolar respecto al grupo control sin MDO, ya que se observa más irregular en los dos grupos experimentales. En el grupo MDO s/USBI se observaron numerosas lagunas de Howship y osteoclastos, con espacios medulares aumentados en su perímetro (Fig. 4).

Análisis Histológico Cuantitativo. Al analizar perímetro vascular y perímetro de espacios medulares, se observaron diferencias cuantitativas entre estas dos variables. Al analizar el perímetro vascular de la zona ligamento periodontaltejido óseo, se obtuvieron diferencias significativas (Kruskal -Wallis $\mathrm{P}<0.008$ ) en la región mesio-coronal (MC) y distoapical (DA) de la raíz mesial con un incremento del perímetro vascular en el grupo MDO c/USBI respecto al grupo control sin MDO (Tabla II). Respecto al perímetro de los espacios medulares óseos, hubo diferencias significativas (Kruskal-Wallis $\mathrm{P}<0.006$ ) entre los grupos control sin MDO y MDO c/USBI, en las regiones MC, MA y DC de la raíz mesial y las regiones MC y DC de la raíz distal. En la región MA de la raíz mesial del grupo MDO c/USBI se observó la 
mayor diferencia respecto de todas las regiones estudiadas (Tabla III). Por último, al analizar la cantidad de osteoclastos del hueso alveolar en relación a la pieza dentaria, no se observaron diferencias significativas entre ellas (Kruskall- Wallis
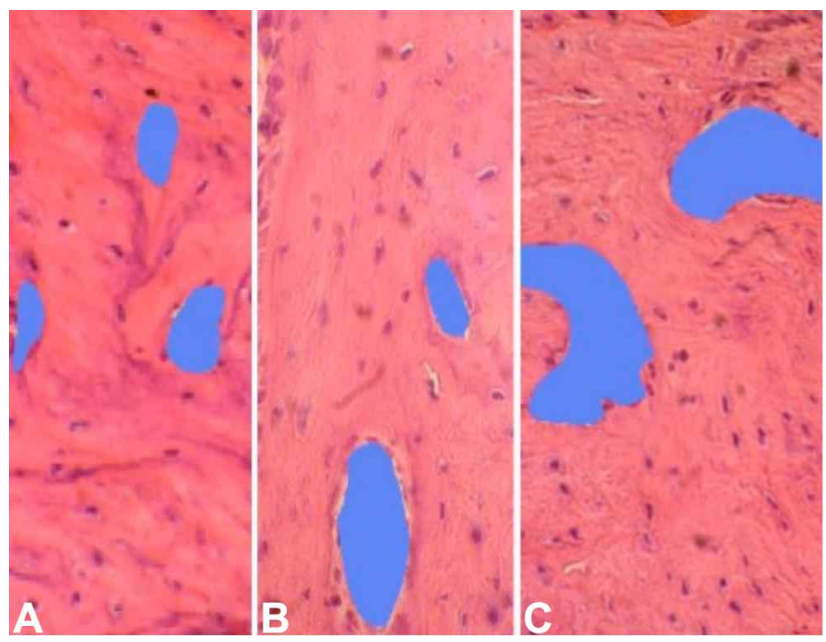

Fig. 3. Perímetro vasos sanguíneos región mesio coronal de raíz mesial. (A) Control sin MDO. (B) MDO s/USBI. (C) MDO c/USBI. p <0.05). Se percibió a su vez que el número de osteoclastos no cambia entre los grupos de estudio al día 21. Sin embargo, se observó que existe una tendencia al aumento de osteoclastos en el grupo MDO s/USBI.
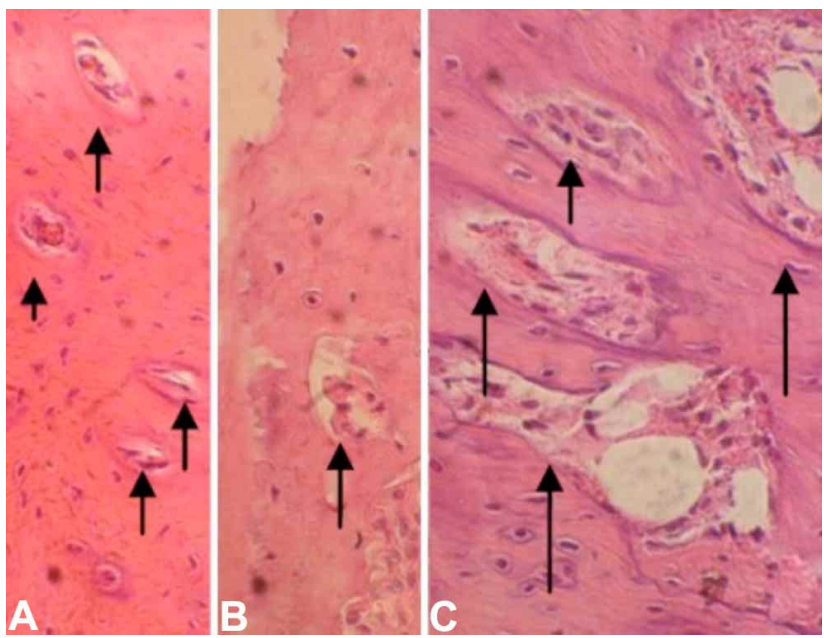

Fig. 4. Perímetro espacios medulares región mesio coronal de raíz mesial (A) Control sin MDO. (B) MDO s/USBI. (C) MDO c/USBI

Tabla II. Diferencias en m del perímetro vascular en las regiones estudiadas entre los distintos grupos de estudio a nivel óseo alveolar.

\begin{tabular}{|c|c|c|c|c|c|c|c|c|c|c|c|c|}
\hline P. Vascular & $\begin{array}{l}\text { RM-MC } \\
\text { media }\end{array}$ & ds & $\begin{array}{l}\text { RM-MA } \\
\text { media }\end{array}$ & $\mathrm{ds}$ & $\begin{array}{l}\text { RM-DC } \\
\text { media }\end{array}$ & ds & $\begin{array}{l}\text { RM-DA } \\
\text { media }\end{array}$ & $\mathrm{ds}$ & $\begin{array}{l}\text { RD-MC } \\
\text { media }\end{array}$ & $\mathrm{ds}$ & $\begin{array}{l}\text { RD-DC } \\
\text { media }\end{array}$ & ds \\
\hline Control sin MDO & 191 & 47 & 245 & 87 & 342 & 154 & 407 & 253 & 438 & 240 & 583 & 165 \\
\hline MDO s/USBI & 284 & 93 & 397 & 148 & 785 & 682 & 672 & 216 & 448 & 28 & 418 & 205 \\
\hline MDO c/USBI & $760 *$ & 119 & 503 & 153 & 672 & 200 & $1079 *$ & 176 & 700 & 380 & 630 & 20 \\
\hline
\end{tabular}

*Diferencias significativas (Kruskall-Wallis $\mathrm{p}<0.008$ ).

Tabla III. Diferencias en $\mathrm{m}$ del perímetro de los espacios medulares en las regiones estudiadas entre los distintos grupos.

\begin{tabular}{|c|c|c|c|c|c|c|c|c|c|c|c|c|}
\hline Espacios medulares & $\begin{array}{l}\text { RM-MC } \\
\text { media }\end{array}$ & ds & $\begin{array}{l}\text { RM-MA } \\
\text { media }\end{array}$ & ds & $\begin{array}{l}\text { RM-DC } \\
\text { media }\end{array}$ & ds & $\begin{array}{l}\text { RM-DA } \\
\text { media }\end{array}$ & ds & $\begin{array}{l}\text { RD-MC } \\
\text { media }\end{array}$ & ds & $\begin{array}{l}\text { RD-DC } \\
\text { media }\end{array}$ & $\mathrm{ds}$ \\
\hline Control sin $\mathrm{MDO}$ & 175 & 50 & 161 & 23 & 290 & 175 & 377 & 160 & 348 & 174 & 512 & 221 \\
\hline MDO s/USBI & 226 & 82 & 233 & 29 & 350 & 7 & 639 & 300 & 743 & 245 & 1164 & 231 \\
\hline MDO c/USBI & $1170 *$ & 202 & $977 *$ & 146 & $1101 *$ & 264 & 740 & 166 & $1552 *$ & 305 & $1444 *$ & 194 \\
\hline
\end{tabular}

*Diferencias significativas (Kruskall-Wallis $\mathrm{p}<0.006$ ).

\section{DISCUSIÓN}

Este estudio fue llevado a cabo mediante un diseño experimental longitudinal controlado, el cual fue ideado para analizar los efectos de la aplicación de USBI en el movimiento dentario ortodóncico en primeros molares superiores de ratas Sprague-Dawley. Se llevó a cabo, utilizando un aparato de Ultrasonido prototipo, el cual emite ondas pulsátiles de baja intensidad.

Se realizó la estimulación de los tejidos dentarios y los tejidos de soporte mediante USBI para observar tanto los efectos en la distancia y velocidad del movimiento dentario, así como también a nivel histológico.

Esto último, con el fin de determinar si es posible acortar el período de tratamiento clínico y estudiar en un nivel histológico, las reacciones tisulares ante la aplicación de fuerzas ortodóncicas.

Los resultados reportados referentes a una significativa aceleración del movimiento dentario ortodóncico, aso- 
ciados a efectos histológicos de distinta índole, permiten confirmar en este modelo experimental que el USBI es una forma de estimulación mecánica celular efectiva. Los resultados obtenidos muestran que en 21 días es posible observar diferencias significativas entre el grupo control, el grupo con aparatos sin estimulación y el grupo con aparatos y estimulación en la magnitud del movimiento dentario, tanto a nivel grupal como entre cada uno de los grupos. Estos resultados coinciden con otros experimentos realizados en ratas aplicando USBI, en los que se observó una mayor cantidad y velocidad de movimiento (Xue et al., 2013; Alazzawi et al., 2018). Sin embargo, contrastan con el estudio de AlDaghreer et al. (2014), quienes en aplicaron USBI en piezas dentarias sometidas a MDO en perros Beagle aplicando USBI, donde la diferencia de cantidad de movimiento dentario entre el lado experimental y el lado control no tuvo diferencia estadísticamente significativa. Dicha discordancia puede deberse a diferencias en el modelo experimental, incluyendo la aparatología y las características del aparato de ultrasonido.

El crecimiento máxilo-mandibular normal en ratas progresa a razón de $0,1 \mathrm{~mm}$ diarios en la porción posterior del maxilar y 0,02 a nivel anterior según Olivera et al. (2003). Estos resultados son consistentes con los obtenidos en nuestro estudio, ya que explican que la leve separación registrada entre el primer molar e incisivos de ratas controles se debe al crecimiento normal del maxilar de las ratas.

El movimiento inicial comienza casi instantáneamente y es un reflejo de deformación tisular, según nuestros resultados este tiene una duración de 4 días en los animales experimentales. La segunda fase representa un retraso en movimiento, que refleja reclutamiento de células y el establecimiento de un microambiente que va a permitir un modelado y remodelado tisular apropiado, acorde a nuestros resultados tuvo una duración de 6 días. La fase final representa un recambio tisular que permite una reducción del estrés aplicado lo que finaliza con la desactivación del aparato (King et al., 1991). Dichas fases pudieron observarse en este estudio para los grupos MDO s/USBI y MDO c/ USBI. A su vez, en presencia de USBI el movimiento siempre progresa a una mayor velocidad, sobre todo en el movimiento inicial. Se ha reportado que los cambios tisulares producto de un tratamiento ortodóncico son más rápidos en ratas que en humanos, aunque sus mecanismos principales serían los mismos (Ren et al., 2004). Esto es consistente con los resultados obtenidos del presente experimento.

Los mayores perímetros vasculares observados en el grupo tratado con USBI pueden deberse al efecto estimulatorio del ultrasonido sobre la angiogénesis. En estudios previos, se ha observado dilatación de los capilares en grupos tratados con USBI (Young \& Dyson, 1990; El-
Bialy et al., 2003), donde se ha concluido que los efectos estimulatorios del ultrasonido pulsátil en la formación ósea se deberían a un aumento en la formación de nuevos vasos sanguíneos. Se ha relacionado la angiogénesis con la formación, remodelación y reparación ósea determinando en particular, que la expresión de factor de crecimiento vascular endotelial (VEGF) hace posible la generación de hueso (Rabie et al., 2001, 2002). El VEGF corresponde a un potente regulador de la neovascularización (El-Bialy et al., 2008), y probablemente este factor haya mediado los cambios observados en la vascularidad de los grupos tratados con USBI.

Se ha reportado que el USBI produce efectos anabólicos en ciertos factores de crecimiento, que resultan finalmente en la diferenciación a células osteogénicas (Qamruddin et al., 2015). A su vez, estudios histológicos realizados por Reitan (1964), demuestran que mientras menor sea la densidad ósea y existan mayor número de espacios medulares, más se facilita la reabsorción ósea. Nuestros resultados plantean que uno de los efectos del USBI fue el incremento de los espacios medulares, lo que se asociaría a una mayor adaptación de las estructuras óseas en las muestras sometidas a fuerzas ortodóncicas, facilitando la aceleración observada en el MDO en las ratas tratadas con USBI.

En este estudio, no se observaron diferencias en el número de osteoclastos entre los tres grupos de estudio. Esto sugiere que no necesariamente la intensidad del fenómeno reabsortivo guarda relación con el número de osteoclastos, y que un número similar de osteoclastos es capaz de generar respuestas diferentes en términos de perímetro de lagunas de reabsorción, esta hipótesis fue planteada de manera similar por Feres et al. (2016), donde demostraron que el uso de USBI, aumenta la actividad osteoclástica, y que esta se relaciona directamente con la cantidad de exposición a USBI. Si bien no se vieron diferencias significativas en la cantidad de osteoclastos, se observó una tendencia de mayor número de estos en el grupo MDO s/USBI. Según los resultados de Alazzawi et al., el USBI sería más eficiente estimulando la actividad osteoblástica que la osteoclástica. Estudios experimentales in vitro utilizando ultrasonido de baja intensidad $(0,068 \mathrm{~W} / \mathrm{cm} 2)$ han demostrado que hay un incremento significativo en el recuento de osteoblastos entre los días 7 -10 de estimulación, mientras que el número de osteoclastos disminuye en forma significativa a partir de esos días (Sun et al., 2001).

El USBI demostró ser un elemento complementario no dañino ni nocivo para los tejidos dentarios y sus tejidos de soporte. Fue posible observar un aumento de la eficacia del tratamiento ortodóncico, indicando que la utilización de USBI sobre el movimiento dentario afecta positivamente la eficiencia y eficacia de dicho proceso. 


\section{CONCLUSIONES}

La aplicación de ondas de Ultrasonido Pulsátil de Baja Intensidad permitió acelerar el movimiento dentario en ratas sin causar efectos clínicos ni histológicos perjudiciales.

El USBI permite aumentar significativamente la velocidad y cantidad del movimiento dentario en ratas. La estimulación de piezas dentarias de ratas con USBI genera un incremento de la angiogénesis y un aumento de los espacios medulares que se asocia a la facilitación del MDO.

ROJAS, V.; NINEHAM, A.; GAJARDO, C.; RODRIGUEZ, A. \& OYONARTE, W. R. Effect of low intensity ultrasound (USBI) in orthodontic dental movement. In vivo study in SpragueDawley rats. Int. J. Morphol., 38(1):101-108, 2020.

SUMMARY: The objective of this study was to analyze the effect of USBI wave application on the speed and distance of dental movement, and its effect at histological level in SpragueDawley rats. In this study twenty-three male Sprague-Dawley rats were randomly divided into three groups. Five rats were used as control without orthodontic tooth movement (MDO), nine rats were subjected to an orthodontic force application without USBI application, and nine were subjected to an orthodontic force application with USBI. The orthodontic forces were performed between the vestibular mesio aspect of the first upper left molar and upper central incisor, pulling the molar mesially, applying an approximate force of 50 grams for 21 days. In light of sedation problems these last two groups were composed of six and eight rats respectively. In the MDO group without USBI, there was a reduction in the space between molars and incisors of $2 \mathrm{~mm}$. In the MDO group with USBI this reduction was $3.4 \mathrm{~mm}$, this difference being statistically significant. Histologically, the MDO c / USBI group showed a vascular perimeter and enlarged spinal spaces with respect to the other groups, however, no significant differences were found in the amount of osteoclasts. It was possible to quantitatively demonstrate that USBI is a form of efficient mechanical stimulation for MDO acceleration over an experimental 21-day period, where significant differences were observed in tooth movement between the control group, the group without USBI and the group with USBI. A greater vascularity was observed in the group with USBI, expressing larger vascular perimeters, which could be due to the stimulatory effect of ultrasound on angiogenesis.

KEY WORDS: Ultrasonic waves; Tooth movement techniques; Sprague-Dawley rats.

\section{REFERENCIAS BIBLIOGRÁFICAS}

Al-Daghreer, S.; Doschak, M.; Sloan, A. J.; Major, P. W.; Heo, G.; Scurtescu, C.; Tsui, Y. \& El-Bialy, T. Effect of low-intensity pulsed ultrasound on orthodontically induced root resorption in beagle dogs. Ultrasound Med. Biol., 40(6):1187-96, 2014.
Alazzawi, M. M. J.; Husein, A.; Alam, M. K.; Hassan, R.; Shaari, R.; Azlina, A. \& Salzihan, M. S. Effect of low level laser and low intensity pulsed ultrasound therapy on bone remodeling during orthodontic tooth movement in rats. Prog. Orthod., 19(1):10, 2018.

Becerra, G. D.; Díaz, Z. J.; Carrión, A. F.; Inostroza, S. C. \& Oyonarte, W. R. Evaluation of mesenchymal stem cell proliferation using different low intensity pulsed ultrasound intensities. Int. J. Morphol., 30(2):6216, 2012.

El-Bialy, T. H.; Elgazzar, R. F.; Megahed, E. E. \& Royston, T. J. Effects of ultrasound modes on mandibular osteodistraction. J. Dent. Res., 87(10):953-7, 2008.

El-Bialy, T.; El-Shamy, I. \& Graber, T. M. Growth modification of the rabbit mandible using therapeutic ultrasound: is it possible to enhance functional appliance results? Angle Orthod., 73(6):631-9, 2003.

El-Bialy, T.; El-Shamy, I. \& Graber, T. M. Repair of orthodontically induced root resorption by ultrasound in humans. Am. J. Orthod. Dentofacial Orthop., 126(2):186-93, 2004.

Feres, M. F. N.; Kucharski, C.; Diar-Bakirly, S. \& El-Bialy, T. Effect of low-intensity pulsed ultrasound on the activity of osteoclasts: An in vitro study. Arch. Oral Biol., 70:73-8, 2016.

Huang, H.; Williams, R. C. \& Kyrkanides, S. Accelerated orthodontic tooth movement: molecular mechanisms. Am. J. Orthod. Dentofacial Orthop., 146(5):620-32, 2014.

Kale, S.; Kocadereli, I.; Atilla, P. \& Asan, E. Comparison of the effects of 1,25 dihydroxycholecalciferol and prostaglandin E2 on orthodontic tooth movement. Am. J. Orthod. Dentofacial Orthop., 125(5):607-14, 2004.

King, G. J.; Keeling, S. D.; McCoy, E. A. \& Ward, T. H. Measuring dental drift and orthodontic tooth movement in response to various initial forces in adult rats. Am. J. Orthod. Dentofacial. Orthop., 99(5):456-65, 1991.

Lu, L. H.; Lee, K.; Imoto, S.; Kyomen, S. \& Tanne, K. Histological and histochemical quantification of root resorption incident to the application of intrusive force to rat molars. Eur. J. Orthod., 21(1):5763, 1999.

Masella, R. S. \& Meister, M. Current concepts in the biology of orthodontic tooth movement. Am. J. Orthod. Dentofacial Orthop., 129(4):458-68, 2006.

Olivera, M. I.; Bozzini, C.; Meta, I. F.; Bozzini, C. E. \& Alippi, R. M. The development of bone mass and bone strength in the mandible of the female rat. Growth Dev. Aging, 67(2):85-93, 2003.

Patil, A. K.; Shetty, A. S.; Setty, S. \& Thakur, S. Understanding the advances in biology of orthodontic tooth movement for improved ortho-perio interdisciplinary approach. J. Indian Soc. Periodontol., 17(3):309-18, 2013.

Proffit, W. Ortodoncia Contemporánea. 5a ed. Madrid, Elsevier, 2013.

Qamruddin, I.; Alam, M. K.; Khamis, M. F. \& Husein, A. Minimally Invasive Techniques to Accelerate the Orthodontic Tooth Movement: A Systematic Review of Animal Studies. Biomed Res. Int., 2015:608530, 2015.

Rabie, A. B.; Shum, L. \& Chayanupatkul, A. VEGF and bone formation in the glenoid fossa during forward mandibular positioning. Am. J. Orthod. Dentofacial Orthop., 122(2):202-9, 2002.

Rabie, A. B.; Zhao, Z.; Shen, G.; Hägg, E. U.; Dr, O. \& Robinson, W. Osteogenesis in the glenoid fossa in response to mandibular advancement. Am. J. Orthod. Dentofacial Orthop., 119(4):390-400, 2001.

Reitan, K. Effects of force magnitude and direction of tooth movement on different alveolar bone types. Angle Orthod., 34(4):244-55, 1964.

Ren, Y.; Maltha, J. C. \& Kuijpers-Jagtman, A. M. The rat as a model for orthodontic tooth movement--a critical review and a proposed solution. Eur. J. Orthod., 26(5):483-90, 2004.

Rutten, S.; Nolte, P. A.; Korstjens, C. M.; van Duin, M. A. \& Klein-Nulend, J. Low-intensity pulsed ultrasound increases bone volume, osteoid thickness and mineral apposition rate in the area of fracture healing in patients with a delayed union of the osteotomized fibula. Bone, 43(2):348-54, 2008. 
Sun, J. S.; Hong, R. C.; Chang, W. H. S.; Chen, L. T.; Lin, F. H. \& Liu, H. C. In vitro effects of low-intensity ultrasound stimulation on the bone cells. J. Biomed. Mater. Res., 57(3):449-56, 2001.

Tyrovola, J. B. \& Spyropoulos, M. N. Effects of drugs and systemic factors on orthodontic treatment. Quintessence Int., 32(5):365-71, 2001.

Xue, H.; Zheng, J.; Cui, Z.; Bai, X.; Li, G.; Zhang, C.; He, S.; Li, W.; Lajud, S. A.; Duan, Y. \& Zhou, H. Low-intensity pulsed ultrasound accelerates tooth movement via activation of the BMP-2 signaling pathway. PLoS One, 8(7):e68926, 2013.

Young, S. R. \& Dyson, M. Effect of therapeutic ultrasound on the healing of full-thickness excised skin lesions. Ultrasonics, 28(3):175-80, 1990.

Zainal Ariffin, S. H.; Yamamoto, Z.; Zainol Abidin, I. Z.; Megat Abdul Wahab, R. \& Zainal Ariffin, Z. Cellular and molecular changes in orthodontic tooth movement. Scientific World J., 11:1788-803, 2011.
Dirección para correspondencia:

Rodrigo Oyonarte Weldt

Departamento de Ortodoncia

Facultad de Odontología

Universidad de los Andes

Monseñor Álvaro del Portillo 12455

Santiago, Las Condes

CHILE

Email: oyonarte@miuandes.cl

Recibido : 08-07-2019

Aceptado: 22-08-2019 